\title{
Assessment of Intraoperative Brain Deformation Using Interventional MR Imaging
}

\author{
Derek L. G. Hill ${ }^{1}$, Calvin R. Maurer,Jr. ${ }^{1,2}$, Alastair J. Martin ${ }^{3,4}$, \\ Saras Sabanathan ${ }^{1}$, Walter A. Hall ${ }^{5}$, David J. Hawkes ${ }^{1}$, \\ Daniel Rueckert ${ }^{1}$, and Charles L. Truwit ${ }^{3}$ \\ 1 Division of Radiological Sciences and Medical Engineering \\ King's College London \\ 5th floor Thomas Guy House, Guy's Hospital, London SE1 9RT, U.K. \\ Derek.Hill@kcl.ac.uk \\ 2 Departments of Neurological Surgery and Biomedical Engineering \\ University of Rochester \\ 601 Elmwood Avenue, Box 670, Rochester, NY 14642, USA \\ 3 Department of Radiology, University of Minnesota, Minneapolis, MN USA \\ 4 Philips Medical Systems, Best, The Netherlands \\ ${ }^{5}$ Department of Neurosurgery, University of Minnesota, Minneapolis, MN, USA
}

\begin{abstract}
We study brain deformation for a series of 8 resection cases carried out in the interventional MR suite at the University of Minnesota. The pattern of deformation is described qualitatively. We also quantify deformation by identifying anatomical landmarks spread over the brain in pre- and post-resection images, and show these values agree well with the results obtained from an automatic non-rigid registration algorithm. For all but one patient, the deformation was significantly greater ipsilateral to the lesion than contralateral, with the contralateral deformation being of the same order as the precision of the measurements. For the remaining patient, there was bi-lateral deformation of several millimetres. Example deformation fields are shown illustrating the distribution of deformation over the brain. The variability of deformation between subjects was considerable, suggesting the automatic correction of intraoperative deformation without use of interventional images may be difficult to achieve.
\end{abstract}

\section{Introduction}

The increasing use of image guided surgery systems for neurosurgery has lead to considerable recent interest in quantifying brain deformation during neurosurgery $[1,3,4,8,7]$. Traditional image guided neurosurgery systems determine the rigid body transformation between pre-operative images and an intraoperative coordinate system (eg: defined by an optical localiser). The intraoperative position of tracked pointers, surgical instruments, microscopes or endoscopes can then be related to pre-operative images. These systems can be very accurate, especially if bone-implanted fiducial markers are used [6], provided the rigid 
Table 1. Details of eight resection patients studied using interventional MR.

\begin{tabular}{|c||r|l|l|l|}
\hline Patient & Age & Gender & lesion position & lesion type \\
\hline \hline 1 & 53 & male & left occipital & lung metastasis \\
\hline 2 & 5 & male & left occipital & astrocytoma with necrosis \\
\hline 3 & 69 & male & left frontal & glioblastoma multiforme \\
\hline 4 & 3 & female & right medial temporal & low grade glioma \\
\hline 5 & 39 & female & right occipital-parietal & meningioma \\
\hline 6 & 41 & female & right frontal & recurrence of oligodendroglioma \\
\hline 7 & 36 & female & superficial left temporal & glioma \\
\hline 8 & 57 & male & left frontal & glioblastoma multiforme \\
\hline
\end{tabular}

body assumption is valid. Any tissue deformation invalidates the rigid body assumption. If the tissue deformation is large compared to the required surgical accuracy, then the overall accuracy of the image guided surgical system will be reduced. Recent studies have shown that the brain surface can deform by $10 \mathrm{~mm}$ or more underneath a craniotomy even before any resection takes place [4]. In many cases, however, the structures of interest, such as the lesion and adjacent blood vessels, are well below the surface of the brain, and these may deform substantially less than the brain surface. Since the recent work quantifying brain deformation has concentrated on surface features $[3,4,8]$, the deformation of the structures of surgical interest remains unknown.

Interventional imaging combined with suitable image analysis software could potentially provide measurements of brain deformation throughout the head, both near and far from the site of resection. Interventional MR is not currently widely available for this application, but the good soft tissue contrast, high resolution in three dimensions and absence of ionising radiation make it ideally suited to this purpose. In this paper we extend our previous preliminary work [7] by reporting measurements of brain deformation taken on eight patients who underwent neurosurgical resections in the University of Minnesota Interventional MR suite. This paper reports results from twice the number of resection cases we previously reported (eight instead of four), provides more quantitative results, and includes results obtained from 3D deformation maps from all cases.

\section{Method}

Eight patients undergoing resective surgery at the University of Minnesota were selected for this study. Their lesions and surgical approaches are listed in table 1.

\subsection{Image Acquisition}

In normal use, the interventional MR scanner acquires small numbers of slices in the vicinity of of the resection site with the patient in the operating position 
immediately prior to craniotomy. Then the patient is re-imaged one or more times during the procedure to check the completeness of the resection. Finally the patient is imaged at the end of the resection. To quantify deformation throughout the brain, we changed the acquisition process to acquire additional sagittal 3D magnetisation prepared rapid gradient echo (MP-RAGE) volume images just before resection, and after resection was complete. These images differ from traditional pre- and post-operative images in that the patient was anaesthetised and lying in the operative position. We refer to them as pre-resection and postresection images.

Gradient echo images have good resolution, and the $5 \mathrm{mT} / \mathrm{m}$ readout-gradient used results in relatively little geometric distortion in the read-out direction caused by B0 inhomogeneity [5]. Gradient echo images are, however, sensitive to susceptibility differences in the object being imaged. In particular, there can be signal loss at the boundary between air and soft tissue. Spin echo images do not have this problem. For patients undergoing resections, the brain is exposed to the air and air can enter the head. The magnitude of any brain deformation could, therefore, be exaggerated by signal loss due to susceptibility artefacts. To establish the extend of this problem we also acquired T2 weighted turbo-spinecho images from some patients.

Images were acquired using a flexible phased array "synergy" coil, which provides good patient access, but produces a less uniform B1 field than a standard bird-cage head coil. As a result, the images acquired in this project have considerable intensity shading.

\subsection{Image Analysis}

We first registered the images to correct for any rigid-body motion between the pre- and post-resection images by maximising the normalised mutual information of the joint probability distribution of the two images [11]. After this rigid alignment difference images were calculated by subtracting the transformed preresection images from the post-resection images. Because many of the images had different intensity gains, the difference image inspected was calculated by iteratively determining an intensity scale value that minimises the mean square difference in voxel intensities.

We subsequently applied a non-rigid registration algorithm to correct any remaining non-rigid motion. This non-rigid registration algorithm uses a twostage transformation model. The first stage captures the global motion of the brain and is modelled by a rigid transformation calculated in the previous step. The second stage captures the local motion of the brain and is modelled by a free-form deformation (FFD) based on B-splines. The non-rigid registration is achieved by maximising the normalised mutual information as a similarity measure between pre- and post-resection images [10]. The algorithm has been previously applied [9] and evaluated for the registration of 3D breast MRI [2]. 


\subsection{Assessment of Intraoperative Deformation}

For each patient, representative 3D points were identified in the frontal, parietal, occipital and temporal lobe of the brain and the lateral ventricles both ipsilateral and contralateral to the lesion. One point was identified in each lobe by a single observer. A point was also marked in the cerebellum. Each point had to be definable in three dimensions, for example a vessel bifurcation, an extrema of curvature of a vessel, or a characteristic 3D feature of a sulcus. The position of the point did, therefore, vary between patients, but for each patient, the same point was identified in both the pre- and post-resection image. The software used to identify points allows the user to measure points on zoomed in images, giving coordinates with sub-voxel precision. The length of the vector separating the two points was calculated as a measure of local brain deformation. To assess reproducibility of the measurements, the points were marked six times each by a single observer. There was a gap of several hours between marking the point on the different occasions. The standard deviations of the local deformation measurements was calculated.

In addition to the assessment of intraoperative brain deformation by an observer, the non-rigid registration algorithm was used to calculate a dense deformation field for the entire brain. To avoid influences of deformable or resected tissue on the calculation of the deformation fields, the post-resection images were segmented using Analyze (Mayo Clinic) and the portion of the image volume corresponding to extra-dural material or resected lesion was excluded from the calculation of the similarity measure during the registration process. The node spacing of the algorithm was set at $15 \mathrm{~mm}$.

\section{Results}

\subsection{Qualitative Description of Deformation}

In many cases the RF inhomogeneity was quite different in the pre- and postresection images, and the amount of contrast agent in the scans was different, so there are many intensity changes between the images that do not correspond to tissue deformation. It is, nevertheless, possible to identify tissue deformation independent of the other intensity effects. The observed deformation is described in table 2. Example difference images from patient 6 is shown in figure 1.

\subsection{Quantitative Assessment of Deformation Using Interactively Identified Points}

The left side of table 3 shows the deformation in different regions of the brain measured by manually marking 3D anatomical points. The standard deviation of the point separations was between 0.1 and $0.9 \mathrm{~mm}$. While the reproducibility of marking the points will vary with the point and patient, these measurements suggest that the precision of the deformation values in table 3 is better than 1.0 $\mathrm{mm}$. 


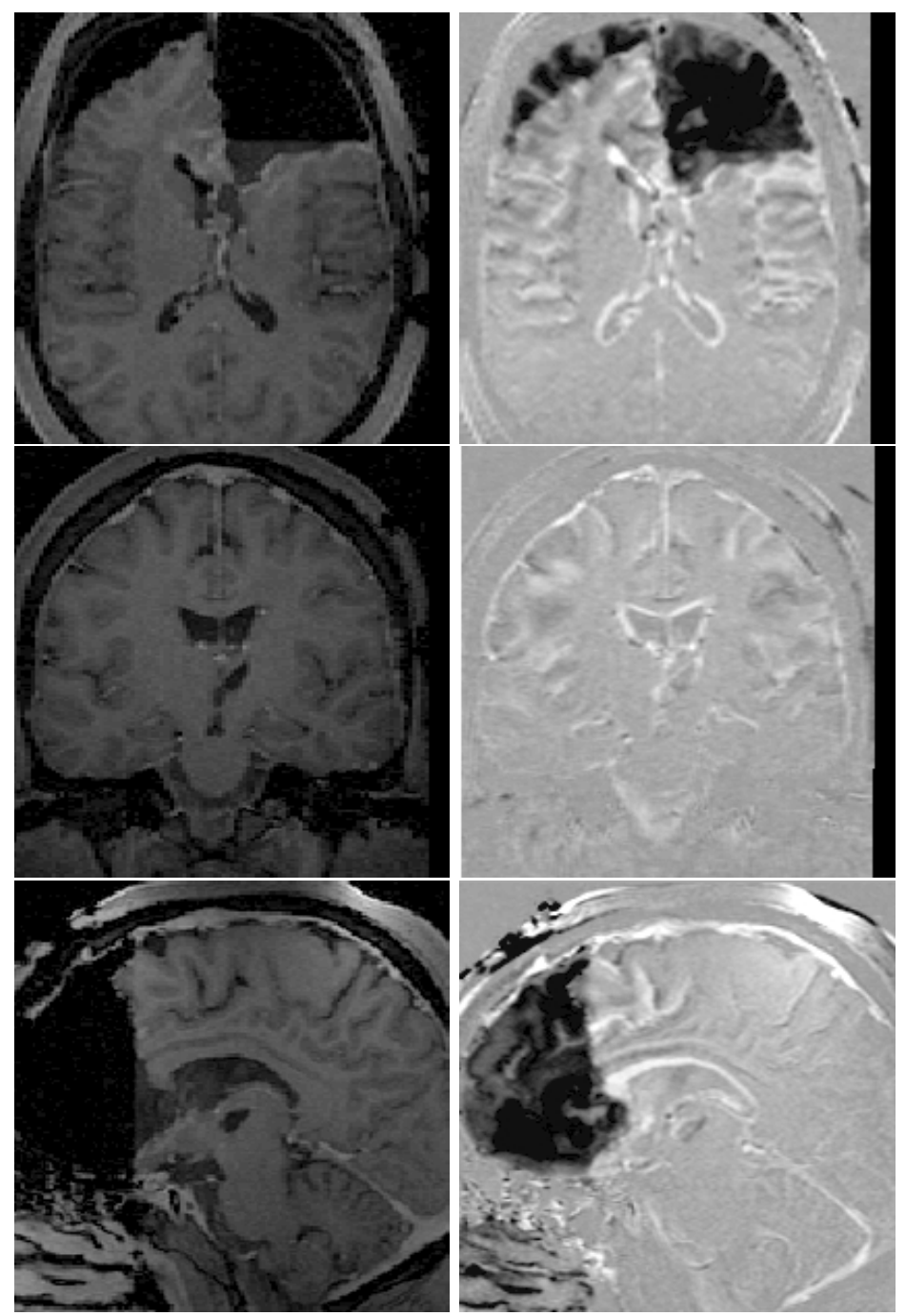

Fig. 1. Example post-resection and difference images from patient 6 . The axial, coronal and sagittal images in the left column are re-formatted from the postresection MP-RAGE volume image. The difference images in the right column are obtained by subtracting the pre-resection image (registered using maximisation of normalised mutual information) from the post-resection image. The dark portions of the frontal lobe indicate resection on the right side and brain deformation alone on the left side side. This was the only patient with substantial bi-lateral deformation. Note also the white line around the lateral ventricles in the difference image: this represents reduction of CSF volume between the preand post-resection images. 
Table 2. Qualitative description of deformation from the eight resection cases listed in table 1 . For each patient, the table shows whether there was shift in the midline or tentorium ( $\mathrm{m} / \mathrm{t}$ shift), and comments on deformation elsewhere.

\begin{tabular}{|c||c|l|}
\hline Patient & $\mathrm{m} / \mathrm{t}$ shift? & Comments \\
\hline \hline 1 & No & Deformation near lesion. Some subtle change in posterior horns \\
\hline 2 & Some & $\begin{array}{l}\text { Substantial deformation near lesion, small volume loss in en- } \\
\text { larged contra-lateral ventricle, small volume gain in ipsilateral } \\
\text { ventricle }\end{array}$ \\
\hline 3 & No & $\begin{array}{l}\text { Deformation near lesion; falling of ipsilateral frontal lobe, subtle } \\
\text { change in anterior horns }\end{array}$ \\
\hline 4 & No & $\begin{array}{l}\text { Deformation at resection site, diffuse ipsilateral gyral deforma- } \\
\text { tion, sinking at entry }(\sim 3 \mathrm{~mm})\end{array}$ \\
\hline 5 & No & $\begin{array}{l}\text { Deformation near lesion. Dark bilateral ventricular rim; little } \\
\text { contralateral cortical deformation }\end{array}$ \\
\hline 7 & No & $\begin{array}{l}\text { Major resection on one side, bilateral brain deformation } \geq 5 \mathrm{~mm} ; \\
\text { Loss of CSF from ventricles and sulci }\end{array}$ \\
\hline 8 & No & $\begin{array}{l}\text { Shift under craniotomy } \geq 5 \mathrm{~mm} ; \text { little contralateral cortical } \\
\text { change; bilateral ventricle volume loss }\end{array}$ \\
\hline \hline
\end{tabular}

\subsection{Deformation Fields}

The non-rigid registration algorithm was run on all eight patients. The algorithm calculates a voxel-by-voxel deformation field. This can be used to transform the pre-resection image into the coordinates of the post-resection image, or it can be used to generate a deformation map, which gives the magnitude and direction of deformation for each voxel. Deformation values calculated by the algorithm for the same points in the brain as used to produce the left side of table 3 are shown in the right side of this same table. $68 \%$ of the values from the deformation map are within $0.5 \mathrm{~mm}$ of the interactively measured values, $90 \%$ are within $1.0 \mathrm{~mm}$, and $95 \%$ are within $1.5 \mathrm{~mm}$. This suggests that the results of the automatic non-rigid registration algorithm agree with the interactive measurements to the precision of the interactive measurements. The values where the agreement is less good tend to be points where the interactively measured deformation is higher.

It is clear from both the interactive and automatic measurements that, with the exception of patient 6 , deformation ipsilateral to the resection is substantially greater than on the contralateral side. A paired Student t-test was used to test whether these differences are significant. Pooling the results for all eight patients, the null hypothesis that the deformation measurements from both sides of the brain come from the same population is rejected $(\mathrm{P}<0.05$ for the interactive measurements; $\mathrm{P}<0.01$ for the automatic algorithm). 
Table 3. Measurement of deformation (in $\mathrm{mm}$ ) in different parts of the brain for eight patients. The left side of the table reports distances between 3D anatomical points interactively identified in the post-resection images and registered preresection images. Reproducibility studies showed that the standard deviation of these measurements varied between $0.1 \mathrm{~mm}$ and $0.9 \mathrm{~mm}$. The right part of the table shows the deformation at these same positions calculated using the free form deformation algorithm described in the text. Entries marked with a - could not be marked in those datasets (eg: that part of the brain had been resected).

\begin{tabular}{|c|c|c|c|c|c|c|c|c|c|c|c|c|c|c|}
\hline \multirow[b]{2}{*}{ Patient } & \multicolumn{7}{|c|}{ Interactive measurements } & \multicolumn{7}{|c|}{ Deformation algorithm values } \\
\hline & 2 & 3 & 4 & 5 & 6 & 7 & 8 & 1 & 3 & 4 & 5 & 6 & 7 & 8 \\
\hline & \multicolumn{14}{|c|}{ Ipsilateral to lesion } \\
\hline $\mathrm{Or}$ & \begin{tabular}{l|l|}
5 & 1.2 \\
\end{tabular} & 2.7 & 2.6 & 0.5 & & 1.0 & 1.0 & \begin{tabular}{|l|l|}
1.0 & 0.5 \\
\end{tabular} & 1.4 & 2.4 & 1.0 & & 1.6 & 0.7 \\
\hline Pari & \begin{tabular}{l|l|}
9 & 7.7 \\
\end{tabular} & 2.2 & 0.8 & 0.7 & 1.7 & & 0.8 & \begin{tabular}{|l|l|}
1.1 & 6.0 \\
\end{tabular} & 0.8 & 0.6 & 0.2 & 2.9 & & 1.0 \\
\hline Tem & - & 0.8 & & 0.7 & 0.7 & & 1.8 & 0.6 & 0.3 & & 0.3 & $\overline{1.3}$ & & 1.8 \\
\hline Occi & 1.5 & 0.6 & 1.0 & 1.6 & 0.2 & 0.6 & 1.7 & 1.3 & 0.3 & 1.2 & 1.9 & 0.5 & 1.0 & 1.0 \\
\hline \multirow[t]{2}{*}{ Lat. ventricle } & \begin{tabular}{|l|l|}
0.6 & 0.7 \\
\end{tabular} & 0.9 & 1.7 & 0.8 & 2.1 & 1.9 & 3.4 & \begin{tabular}{|l|l|}
0.4 & 0.7 \\
\end{tabular} & 0.5 & 1.0 & 0.3 & 0.5 & & 3.1 \\
\hline & \multicolumn{14}{|c|}{ Contralateral to lesion } \\
\hline be & 0.5 & 0.5 & 0.8 & 0.7 & 6.0 & 0.7 & 0.6 & \begin{tabular}{|l|l|}
0.6 & 0.5 \\
\end{tabular} & 0.5 & 0.5 & 0.8 & 6.8 & 0.5 & 0.6 \\
\hline l lobe & \begin{tabular}{|l|l|}
1.8 & 0.9 \\
\end{tabular} & 0.5 & 0.6 & 0.6 & 0.7 & 0.6 & 1.0 & \begin{tabular}{|l|l|}
0.6 & 1.3 \\
\end{tabular} & 0.5 & 0.5 & 0.5 & 0.4 & 0 . & 1. \\
\hline ral lobe & \begin{tabular}{|l|l|}
0.5 & 0.6 \\
\end{tabular} & 0.4 & 1.0 & 0.5 & 0.5 & 0.4 & 0.8 & \begin{tabular}{|l|l|}
0.2 & 0.3 \\
\end{tabular} & 1.0 & \begin{tabular}{|l|}
0.3 \\
\end{tabular} & 0.0 & \begin{tabular}{|l|}
0.8 \\
\end{tabular} & 0.3 & 0.5 \\
\hline al lobe & \begin{tabular}{|l|l|}
0.5 & 2.5 \\
\end{tabular} & 1.0 & 0.7 & 0.5 & 0.5 & 0.6 & 0.7 & \begin{tabular}{|l|l|}
0.3 & 0.3 \\
\end{tabular} & 0.2 & 0.3 & 0.1 & 0.6 & 0.4 & 0.3 \\
\hline ntricle & \begin{tabular}{|l|l|}
0.9 & 1.2 \\
\end{tabular} & 1.0 & 1.0 & 1.3 & 5.0 & 1.1 & 0.6 & \begin{tabular}{|l|l|}
0.1 & 0.3 \\
\end{tabular} & 0.3 & 0.3 & 0.3 & \begin{tabular}{|l|}
6.0 \\
\end{tabular} & 0.8 & 0.9 \\
\hline llum & \begin{tabular}{|l|l|}
2.2 & 0.6 \\
\end{tabular} & 0.5 & 09 & 0.6 & 0.9 & 05 & 0.2 & \begin{tabular}{|l|l|}
0.3 & 0.5 \\
\end{tabular} & 0.2 & 0.2 & 0.2 & \begin{tabular}{|l|l|}
1.0 \\
\end{tabular} & $\overline{03}$ & 0.3 \\
\hline
\end{tabular}

All the resections were supra-tentorial. It is noticeable that the deformation of the cerebellum was $1 \mathrm{~mm}$ or less for all cases according to the automatic algorithm, and for all but one case, according to the interactive measurements.

Figure 2 compares difference images obtained by subtracting the transformed pre-resection image from the post-resection image for patient 4 . Reformated axial and sagittal slices are shown as these clearly illustrate the deformation below the craniotomy. It is clear from the difference images that transformation using the non-rigid registration algorithm results in better alignment of pre- and postresection images than using the rigid-body registration algorithm. Figure 3 shows the region around the craniotomy of patient 4 in more detail. The deformation map overlaid on the post-resection image indicates that the deformation corresponding to a falling of the brain away from the craniotomy, in approximately the direction of gravity. This deformation is greatest immediately beneath the craniotomy, and by the time the mid-line is reached, becomes virtually zero. This figure also compares the overlay of the pre-resection image on the post-resection image using the rigid body transformation and deformation field shown. There is much better alignment of both brain surface and ventricles using the deformation algorithm. 

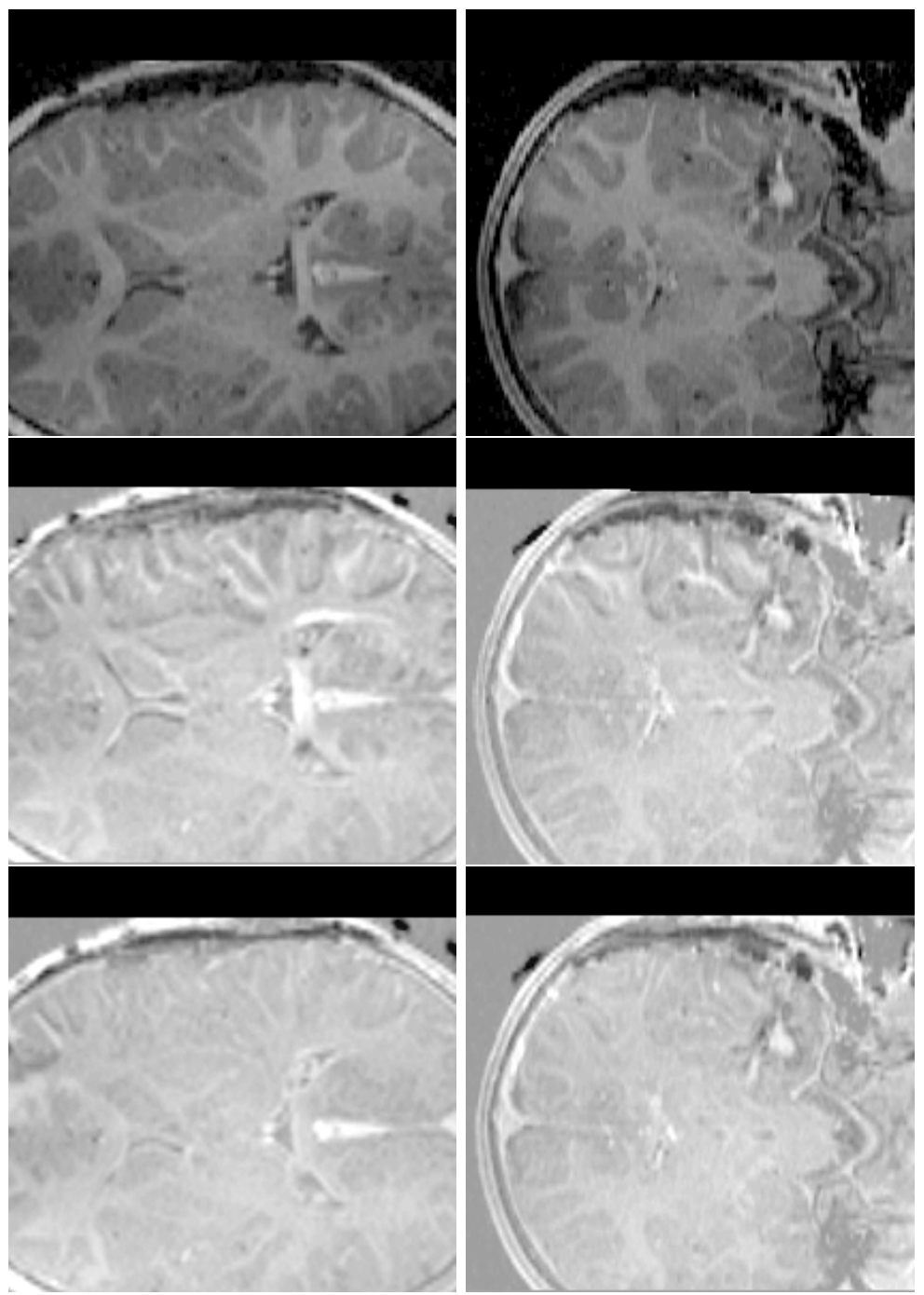

Fig. 2. Example coronal and axial slices from patient 4, showing the transformed pre-resection image subtracted from the post-resection image. The top row shows the post resection images, the middle row the subtraction image produced after rigid body registration, and the bottom row the subtraction image after nonrigid registration. Notice the reduction in residual signal in the subtraction image using free form deformation. The residual difference after free form deformation results from contrast in the post-resection image and differences in RF inhomogeneity, as well as uncorrected tissue deformation. The slices are orientated such that the gravitationally most apendent part of the patient is at the top of the page. 


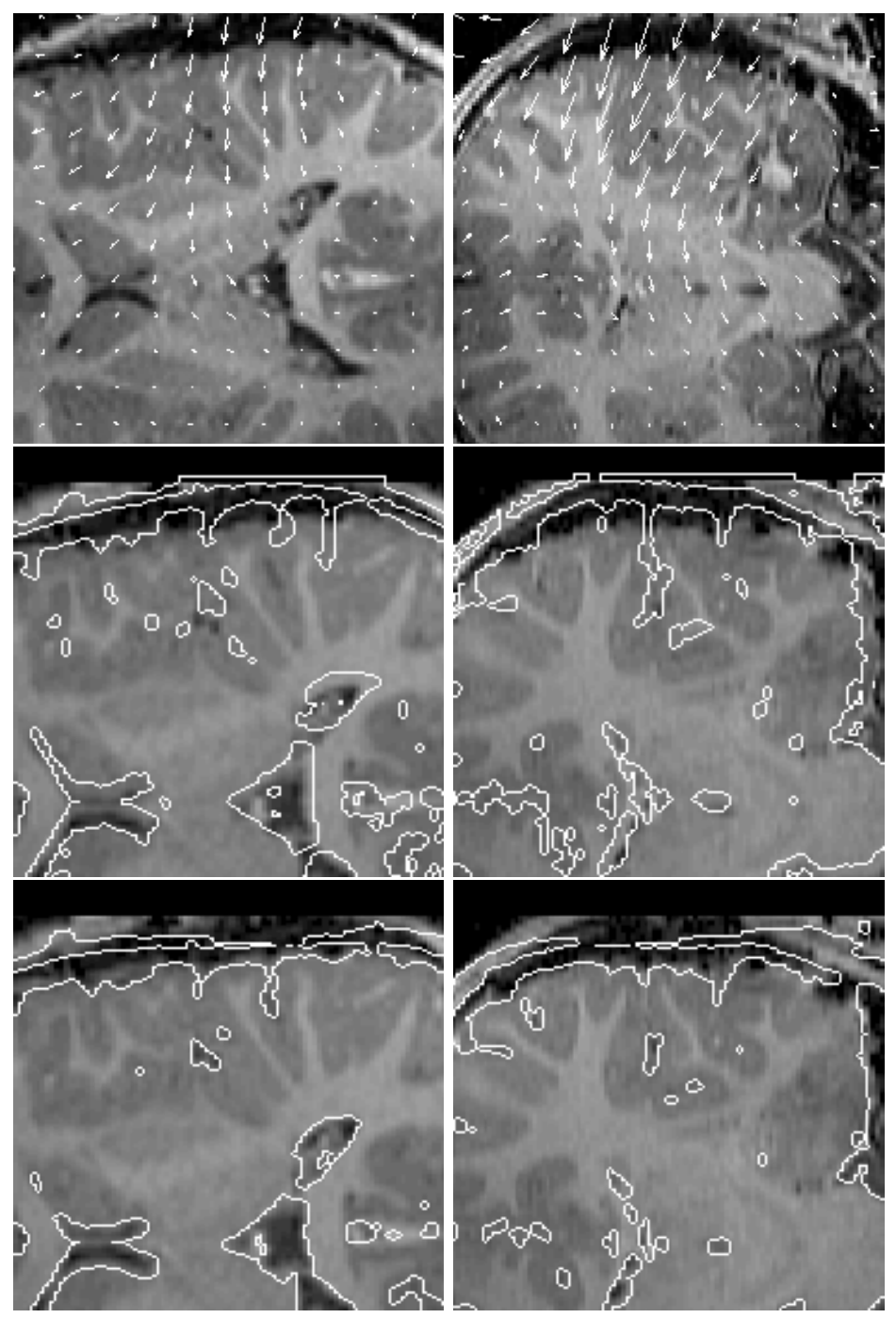

Fig. 3. Brain deformation in the vicinity of the craniotomy for patient 4 . All rows show axial and coronal views approximately aligned with the patient's intraoperative position. The top row shows the deformation field overlaid as white arrows on the post-resection image. The length of the vectors have been exagerated for visualization purposes. The middle and bottom row show the boundary of the pre-resection volume overlaid on the post-resection volume using the rigid body (middle) and free-form deformation (bottom) transformation respectively. The free-form deformation algorithm results in better alignment of both the cortical surface and ventricle outlines than the rigid body transformation. 


\section{Discussion}

We have assessed intraoperative brain deformation on eight patients who underwent neurosurgical resections in the interventional MR suite at the University of Minnesota. We have described the deformation qualitatively, and quantified the deformation over the brain volume using interactively identified anatomical landmarks. We have also used a non-rigid registration algorithm to automatically calculate a deformation field that gives a deformation magnitude and direction for each voxel in the image. The algorithm used to calculate the deformation field is a modification of an algorithm previously used to non-rigidly register pre- and post-contrast MR mammograms. The algorithm provides deformation values that agree well with interactively measured deformation for all patients. We believe that this non-rigid registration algorithm is useful both for quantifying brain deformation in this on-going study, and is also likely to provide a method for overlaying information from pre-operatively acquired images from MR or other modalities on interventional images. This could, for example, be used to overlay a pre-operative surgical plan, or features of interest onto intraoperatively acquired MR images.

The intraoperative brain deformation for this set of eight patients was quite variable. In some, but not all cases, there was no shift in the midline or tentorium. Similarly, in some, but not all cases, there was very little deformation contralateral to the lesion. Also, the magnitude of deformation ipsilateral to the resection, but away from the site of surgery was variable. This suggests that the mechanisms of brain deformation are likely to be quite complex. Simple physical models of brain deformation may, therefore, be inadequate for compensating for brain deformation in image guided surgery systems that do not make use of intraoperative imaging.

\section{Acknowledgements}

We thank Frans Gerritsen and Joop van Vaals of Philips Medical Systems for assistance and the UK EPSRC (GR/M47294 and GR/L08519) and the EU (EASI project HC1012 under 4th framework programme) for financial support.

\section{References}

1. R. D. Bucholz, D. D. Yeh, J. Trobaugh, L. L. McDurmont, C. D. Sturm, C. Baumann, J. M. Henderson, A. Levy, and P. Kessman, "The correction of stereotactic inaccuracy caused by brain shift using an intraoperative ultrasound device", in CVRMed-MRCAS '97, J. Troccaz, E. Grimson, and R. Mösges, Eds., pp. 459-466. Springer-Verlag, Berlin, 1997. 910

2. E. R. E. Denton, L. I. Sonoda, D. Rueckert, S. C. Rankin, C. Hayes, M. Leach, D. L. G. Hill, and D. J. Hawkes, "Comparison and evaluation of rigid and non-rigid registration of breast MR images", J. Comput. Assist. Tomogr., 1999, In press. 912 
3. N. L. Dorward, O. Alberti, B. Velani, F. A. Gerritsen, W. F. J. Harkness, N. D. Kitchen, and D. G. T. Thomas, "Postimaging brain distortion: Magnitude, correlates, and impact on neuronavigation", J. Neurosurg., vol. 88, pp. 656-662, 1998. 910, 911

4. D. L. G. Hill, C. R. Maurer, Jr., R. J. Maciunas, J. A. Barwise, J. M. Fitzpatrick, and M. Y. Wang, "Measurement of intraoperative brain surface deformation under a craniotomy", Neurosurgery, vol. 43, pp. 514-526, 1998. 910, 911

5. D. L. G. Hill, C. R. Maurer, Jr., C. Studholme, J. M. Fitzpatrick, and D. J. Hawkes, "Correcting scaling errors in tomographic images using a nine degree of freedom registration algorithm", J. Comput. Assist. Tomogr., vol. 22, pp. 317-323, 1998. 912

6. C. R. Maurer, Jr., J. M. Fitzpatrick, M. Y. Wang, R. L. Galloway, Jr., R. J. Maciunas, and G. S. Allen, "Registration of head volume images using implantable fiducial markers", IEEE Trans. Med. Imaging, vol. 16, pp. 447-462, 1997. 910

7. C. R. Maurer, Jr., D. L. G. Hill, A. J. Martin, H. Liu, M. McCue, D. Rueckert, D. Lloret, W. A. Hall, R. E. Maxwell, D. J. Hawkes, and C. L. Truwit, "Investigation of intraoperative brain deformation using a 1.5 Tesla interventional MR system: Preliminary results", IEEE Trans. Med. Imaging, vol. 17, pp. 817-825, 1998. 910, 911

8. D. W. Roberts, A. Hartov, F. E. Kennedy, M. I. Miga, and K. D. Paulsen, "Intraoperative brain shift and deformation: a quantitative analysis of cortical displacement in 28 cases", Neurosurgery, vol. 43, pp. 749-758, 1998. 910, 911

9. D. Rueckert, C. Hayes, C. Studholme, P. Summers, M. Leach, and D. J. Hawkes, "Non-rigid registration of breast MR images using mutual information", in First Int. Conf on Medical Image Computing and Computer-Assisted Intervention (MICCAI '98), Cambridge, MA, 1998, pp. 1144-1152. 912

10. D. Rueckert, L. I. Sonoda, C. Hayes, D. L. G. Hill, M. O. Leach, and D. J. Hawkes, "Non-rigid registration using free-form deformations: Application to breast MR images", IEEE Trans. Med. Imaging, 1999, In Press. 912

11. C. Studholme, D. L. G. Hill, and D. J. Hawkes, "An overlap invariant entropy measure of 3D medical image alignment", Pattern Recognition, vol. 32, pp. 71-86, 1999. 912 